$\underline{\text { Preprint typeset in JHEP style. - HYPER VERSION }}$

SWAT/309

\title{
Cosmological Brane World Solutions with Bulk Scalar Fields
}

\author{
Stephen C. Davis \\ Department of Physics, University of Wales Swansea, \\ Singleton Park, Swansea, SA2 8PP, Wales \\ E-mail: S.C.Davis@swansea.ac.uk
}

\begin{abstract}
Cosmological brane world solutions are found for five-dimensional bulk spacetimes with a scalar field. A supergravity inspired method for obtaining static solutions is combined with a method for finding brane cosmologies with constant bulk energies. This provides a way to generate full (bulk and brane) cosmological solutions to brane worlds with bulk scalar fields. Examples of these solutions, and their corresponding Friedmann equations, are discussed.
\end{abstract}

Keywords: Extra Large Dimensions, Physics of the Early Universe, Cosmology of Theories beyond the SM, Supergravity Models. 


\section{Introduction}

There has been considerable interest in the idea that our universe may be a 3-brane embedded in a higher dimensional bulk space. This possibility has been motivated by ideas in string/M-theory. All the usual standard model fields are confined to the brane, while gravity can propagate in the bulk. So far, gravity experiments have not detected any higher dimensional effects, so if this 'brane world' scenario is to be a model of our universe, they must be suppressed. In a scenario considered by Randall and Sundrum [1], a positive tension 3-brane is embedded in a five-dimensional bulk space with a negative cosmological constant. If the brane tension and bulk energy density are chosen appropriately, the standard four-dimensional gravity will arise from graviton bound states on the brane. The gravitons which are free to propagate in the bulk give corrections to this.

This idea was extended to an evolving universe by Binétruy, Deffayet, Ellwanger and Langlois [2, 3]. As well as the usual brane energy density there is also a brane cosmological constant. If this is chosen appropriately it will cancel the bulk energy's contribution on the brane and give a cosmology whose late time Friedmann equation is approximately the same as in the standard Friedmann-Robertson-Walker universe. Thus the brane cosmology predictions for the current evolution of the universe are as normal. However, the initial evolution of the universe is dominated by the nonstandard terms arising from brane effects.

In the Randall-Sundrum brane world scenario the bulk contains only a cosmological constant. String/M-theory suggests that it will also contain scalar fields, which are free to propagate through the bulk [4]. The evolution of these fields may have interesting cosmological effects. For example, if the bulk energy density varies with time, the effective brane cosmological constant will also be time-dependent. This could give alternative mechanisms for inflation and quintessence.

Solving the field equations for a brane world cosmology with scalar fields is far more complicated than in the constant bulk case. One approach is to express the five-dimensional Einstein equations in terms of four-dimensional tensors on the brane [5], and then use boundary conditions to determine them [6]. Unfortunately this approach does not fully determine all the tensors. Progress can be made by making assumptions about the bulk solution [6, 7, 8], but it is not obvious that such assumptions are justified, or self-consistent. To determine which solutions of the four-dimensional Einstein equations are allowed, the full five-dimensional theory must be solved.

Full bulk solutions for static brane worlds have been found in some special cases. If the scalar potential takes a supergravity-like form, the field equations can be reduced to first order equations, which can be solved with relative ease [9, 10]. In this paper I will combine that method with the work of Binétruy et al. [3] to give cosmological solutions. 
Conventions and background are outlined in section 2. In section 3 I extend the results of ref. [3] to a special class of brane world cosmologies with bulk scalar fields. In section the supergravity inspired static solution generating technique is generalised to these brane cosmologies. Some examples of this type of solution are considered in section 5, and their bulk behaviour is discussed in section 6. The results are summarised in section 7 .

\section{Five-dimensional Brane Worlds}

We will consider a theory with the effective five-dimensional action

$$
S=\int_{\text {bulk }} d^{5} x \sqrt{-g}\left\{\frac{1}{2 \kappa^{2}} R-\frac{1}{2}(\nabla \phi)^{2}-V(\phi)\right\}-\int_{\text {brane }} d^{4} x \sqrt{-h}\left\{\frac{1}{\kappa^{2}}[K]+\mathcal{L}_{\mathrm{b}}(\phi)\right\} .
$$

$g_{A B}$ is the bulk metric, and $h_{A B}$ is the induced metric on the brane. They are related by $h_{A B}=g_{A B}-N_{A} N_{B}$, where $N^{A}$ is the unit vector normal to the 3 -brane. The covariant derivatives corresponding to $g_{A B}$ and $h_{A B}$ are $\nabla_{A}$ and $D_{A}$ respectively. The Gibbons-Hawking boundary term, $[K]$, is required to give a well-formulated action. $[K]$ is the change in $K=h^{A B} K_{A B}$ across the brane, and $K_{A B}=h_{A}^{C} h_{B}^{D} \nabla_{(C} N_{D)}$ is the extrinsic curvature. $\kappa^{2}=8 \pi / M_{5}^{3}$, where $M_{5}$ is fundamental five-dimensional Planck mass. $\mathcal{L}_{\mathrm{b}}(\phi)$ is the Lagrangian density for all the fields on the brane. It includes the 'ordinary' standard model fields which are confined to the brane, and the brane potential energy for the bulk scalar $\phi$.

Varying the action gives the bulk equations of motion

$$
\begin{gathered}
\nabla^{2} \phi=\frac{d V}{d \phi} \\
G_{B}^{A}=\kappa^{2} T_{B}^{A}=\kappa^{2}\left(\nabla^{A} \phi \nabla_{B} \phi-\delta_{B}^{A}\left[\frac{1}{2}(\nabla \phi)^{2}+V(\phi)\right]\right) .
\end{gathered}
$$

The brane part of the action gives the jump conditions [4]

$$
\begin{gathered}
{\left[N^{A} \nabla_{A} \phi\right]=\frac{\delta \mathcal{L}_{\mathrm{b}}}{\delta \phi},} \\
{\left[K_{A B}-K h_{A B}\right]=-\kappa^{2} t_{A B},}
\end{gathered}
$$

where $t_{A B}$ is the energy momentum tensor corresponding to $\mathcal{L}_{\mathrm{b}}(\phi)$.

The Codacci equation relates a projection of $G_{A B}$ to $K_{A B}$

$$
N^{A} G_{A B} h_{C}^{B}=D_{A} K_{C}^{A}-D_{C} K
$$

Combining this with (2.3) and substituting it into the jump conditions (2.4) and (2.5) implies

$$
D_{A} t_{C}^{A}=-\frac{\delta \mathcal{L}_{\mathrm{b}}}{\delta \phi} D_{C} \phi
$$


This is the effective energy-momentum conservation equation on the brane.

Following ref. [2] we consider a five-dimensional metric of the form

$$
d s^{2}=g_{A B} d x^{A} d x^{B}=-n^{2}(t, y) d t^{2}+a^{2}(t, y) \Omega_{i j} d x^{i} d x^{j}+b^{2}(t, y) d y^{2}
$$

where $\Omega_{i j}$ is a three-dimensional metric of space with constant curvature $k=-1,0,1$. The coordinates along the brane are denoted $x^{\mu}$ and $y=x^{5}$ is the fifth bulk coordinate. The brane is located at $y=0$. Differentiation with respect to $y$ and $t$ will be denoted by primes and dots respectively. The brane's normal is $N^{A}=\delta_{5}^{A} b^{-1}$, and the extrinsic curvature is $K_{B}^{A}=\operatorname{diag}\left(n^{\prime} /(n b), \delta_{j}^{i} a^{\prime} /(a b), 0\right)$.

Assuming that the brane matter is describable by a perfect fluid, $t_{\nu}^{\mu}=\operatorname{diag}\left(-\rho_{\mathrm{b}}, p_{\mathrm{b}}, p_{\mathrm{b}}, p_{\mathrm{b}}\right)$. The jump conditions (2.4) and (2.5) are then

$$
\begin{gathered}
\frac{\left[\phi^{\prime}\right]}{b_{0}}=\frac{\delta \mathcal{L}_{\mathrm{b}}}{\delta \phi}, \\
\frac{\left[a^{\prime}\right]}{a_{0} b_{0}}=-\frac{\kappa^{2}}{3} \rho_{\mathrm{b}}, \\
\frac{\left[n^{\prime}\right]}{a_{0} b_{0}}=\frac{\kappa^{2}}{3}\left(3 p_{\mathrm{b}}+2 \rho_{\mathrm{b}}\right),
\end{gathered}
$$

The subscript 0 denotes quantities at $y=0$, i.e. on the brane, and $[Q]=Q\left(y=0^{+}\right)-$ $Q\left(y=0^{-}\right)$is the change in $Q$ across the brane. If we assume that the bulk solution is symmetric across the brane, i.e. invariant under $y \leftrightarrow-y$, then $Q\left(0^{-}\right)=-Q\left(0^{+}\right)$ and (2.9 2.11) can be used to determine $\phi^{\prime}, a^{\prime}$ and $n^{\prime}$ at $y=0$.

The energy-momentum conservation equation (2.7) becomes

$$
\dot{\rho}_{\mathrm{b}}+3 \frac{\dot{a}_{0}}{a_{0}}\left(\rho_{\mathrm{b}}+p_{\mathrm{b}}\right)=\frac{\delta \mathcal{L}_{\mathrm{b}}}{\delta \phi} \dot{\phi}_{0}
$$

The right-hand side of this equation gives the amount of energy non-conservation of the matter fields on the brane.

\section{Brane Cosmology with Scalar Fields}

Using the Gauss-Codacci formalism it is possible to express the five-dimensional Einstein equations in terms of the effective four-dimensional Einstein tensor, the extrinsic curvature, and the electric part of the Weyl tensor [5]. Using the jump conditions the extrinsic curvature is found in terms of the brane energy-momentum. The Weyl tensor cannot be found in this way, so another approach is needed to find full bulk solutions. I will consider a special class of solutions for which the fivedimensional field equations can be simplified and solved using generalisations of the methods of refs. [3] and [10]. 
In order to simplify the Einstein equations, it is useful to introduce the quantity

$$
\mathcal{F}(t, y)=-\left(\frac{\dot{a}}{a n}\right)^{2}+\left(\frac{a^{\prime}}{a b}\right)^{2}
$$

In the bulk the components of the Einstein tensor are related to $\mathcal{F}$ by

$$
\begin{aligned}
G_{0}^{0}-\frac{\dot{a}}{a^{\prime}} G_{5}^{0} & =\frac{3}{2 a^{3} a^{\prime}} \partial_{y}\left(a^{4} \mathcal{F}\right)-\frac{3 k}{a^{2}} \\
G^{5}{ }_{5}-\frac{a^{\prime}}{\dot{a}} G^{5}{ }_{0} & =\frac{3}{2 a^{3} \dot{a}} \partial_{t}\left(a^{4} \mathcal{F}\right)-\frac{3 k}{a^{2}} .
\end{aligned}
$$

This extends a simplification used in ref. [3]. In that paper the bulk energy was taken to be constant. The left hand sides of (3.2) and (3.3) are then equal, and it is simple to show that they imply $\mathcal{F}=\mathcal{F}(a)$. The Einstein equations can then be solved with relative ease.

When scalar fields are introduced it is clear, after substituting in (2.3), that the left hand sides of (3.2) and (3.3) are not generally equal. However if we consider a special class of solutions with $\phi=\phi(a)$, the two expressions will be the same. Hence, as with the constant bulk case, $\mathcal{F}^{\prime} / a^{\prime}=\dot{\mathcal{F}} / \dot{a}$, and so $\mathcal{F}=\mathcal{F}(a)$ too. Both (3.2) and (3.3) then reduce to

$$
a \frac{d \mathcal{F}}{d a}+4 \mathcal{F}+\frac{\kappa^{2}}{3} \mathcal{F}\left(a \frac{d \phi}{d a}\right)^{2}+2 \frac{\kappa^{2}}{3} V(\phi)-\frac{2 k}{a^{2}}=0 .
$$

The other diagonal components of the Einstein equation $\left(G_{i j}=\kappa^{2} T_{i j}\right)$ are automatically satisfied as a result of the Bianchi identities.

The standard brane cosmology discussed in ref. [3] is a simple example of this type of solution. It has $\phi=$ const., $V=$ const. $<0$ and

$$
\mathcal{F}=-\frac{\kappa^{2}}{6} V+\frac{k}{a^{2}}-\frac{\mathcal{C}}{a^{4}} .
$$

where $\mathcal{C}$ is an arbitrary integration constant.

On the brane the effective four-dimensional Friedmann equation can be determined from $\mathcal{F}$. Assuming the $Z_{2}$ symmetry, (3.1) and (2.10) imply

$$
\frac{\dot{a}_{0}^{2}}{a_{0}^{2}}=\frac{\kappa^{4}}{36} \rho_{\mathrm{b}}^{2}-\mathcal{F}_{0} .
$$

The time has been scaled so that $n_{0}=1 . t$ is thus the proper cosmological time on the brane.

When $\phi=\phi(a)$, the field equation (2.2) reduces to

$$
\nabla^{2} \phi=a^{2} \mathcal{F}\left(\frac{d^{2} \phi}{d a^{2}}+\frac{1}{a} \frac{d \phi}{d a}\right)+\left[\left(G_{0}^{0}+G^{5}{ }_{5}\right) \frac{a}{3}+\frac{2 k}{a}\right] \frac{d \phi}{d a}=\frac{d V}{d \phi} .
$$


Substituting $G_{A B}=\kappa^{2} T_{A B}$ and (3.4) into (3.7) gives

$$
\mathcal{F}\left(a \frac{d}{d a}\right)^{2} \phi+\left[a \frac{d \mathcal{F}}{d a}+4 \mathcal{F}+\frac{\kappa^{2}}{3} \mathcal{F}\left(a \frac{d \phi}{d a}\right)^{2}\right] a \frac{d \phi}{d a}-\frac{d V}{d \phi}=0
$$

Thus the original partial differential field equations have been reduced to two ordinary differential equations.

\section{Supergravity-style Solutions}

For certain choices of $V$ it is possible to generate solutions to the field equations using simpler first order equations. If $k=0$ and $V$ is a supergravity-style potential

$$
V(\phi)=\frac{1}{8}\left(\frac{d W}{d \phi}\right)^{2}-\frac{\kappa^{2}}{6} W^{2}
$$

then the field equations (3.4) and (3.8) are satisfied if

$$
\begin{gathered}
\mathcal{F}=\frac{\kappa^{4}}{36} W^{2} \\
a \frac{d \phi}{d a}=-\frac{3}{\kappa^{2} W} \frac{d W}{d \phi} .
\end{gathered}
$$

These equations are a generalisation of those discussed in refs. [9] and [10, where static ( $t$ independent) brane worlds were considered. The corresponding first order equations discussed in those papers can be obtained by setting $b=1, n=a=e^{A(y)}$.

If we were dealing with a supergravity theory then $W(\phi)$ would be the superpotential. However supergravity is not required for solutions of (4.1 4.3) to satisfy (3.4) and (3.8). All that is needed is the above form of the potential. For conve-

nience I will refer to $W$ as the superpotential whether the underlying theory includes supergravity or not.

The Friedmann equation (3.6) becomes

$$
\frac{\dot{a}_{0}^{2}}{a_{0}^{2}}=\frac{\kappa^{4}}{36}\left[\rho_{\mathrm{b}}^{2}-W_{0}^{2}\right]
$$

Using (4.3) the rate of change of $\phi$ on the brane can also be found.

$$
\dot{\phi}_{0}^{2}=\frac{1}{4}\left(\frac{d W_{0}}{d \phi}\right)^{2}\left[\frac{\rho_{\mathrm{b}}^{2}}{W_{0}^{2}}-1\right] .
$$

The equation (4.3) relates the two jump conditions (2.9) and (2.10). If the bulk is $Z_{2}$ symmetric, the jump conditions are only consistent if

$$
\frac{\delta \mathcal{L}_{\mathrm{b}}}{\delta \phi}=\left(\frac{1}{W} \frac{d W}{d \phi}\right)_{0} \rho_{\mathrm{b}} .
$$


Thus the energy on the brane is not conserved in this type of brane cosmology. In order for $\mathcal{L}_{\mathrm{b}}$ is to satisfy the above condition, there must be $\phi$ dependent couplings in the standard model Lagrangian. Some of the models discussed in ref. [6] (or generalisations of them) may be suitable.

Combining (4.6) with (2.12) gives the new energy conservation equation

$$
\dot{\rho}_{\mathrm{b}}+3 \frac{\dot{a}_{0}}{a_{0}}\left(\rho_{\mathrm{b}}+p_{\mathrm{b}}\right)=\frac{\dot{W}_{0}}{W_{0}} \rho_{\mathrm{b}} .
$$

Thus if $\dot{W}_{0} / W_{0}$ is negative, energy will leak off the brane. If it is positive, energy will be pushed out of the bulk and onto the brane.

In the usual brane cosmology, the energy density $\rho_{\mathrm{b}}$ consists of a cosmological constant part and an ordinary matter part. The simplest generalisation of this is

$$
\rho_{\mathrm{b}}=\lambda W_{0}+W_{0} \rho
$$

where $\rho$ is proportional to the energy density of the ordinary matter. It has the same evolution as in the standard cosmology, i.e. $\rho \propto a^{-3(1+w)}$ if $p=w \rho$. More complicated solutions of (4.7) will also exist, but I will not consider them here.

The effective Friedmann equation (4.4) then becomes

$$
\frac{\dot{a}_{0}^{2}}{a_{0}^{2}}=\frac{\kappa^{4}}{36} W_{0}^{2}\left[\left(\lambda^{2}-1\right)+\rho^{2}+2 \lambda \rho\right],
$$

It resembles the usual brane cosmology, but with a time dependent gravitational coupling strength. If $W$ tends to a suitable constant at large $t$ then a conventional brane world Friedmann equation will be obtained. If $\lambda=1$ this will give the same late time evolution as the standard cosmology, i.e. $\dot{a}_{0}^{2} / a_{0}^{2} \propto \rho$.

By considering the brane Friedmann equation (4.9), we can rule out many choices of $W$. If $W$ does not tend to a constant, then the solution's cosmological evolution will not generally be compatible with observations. It is still possible that such solutions will agree with the standard cosmology at some intermediate time. However, we expect the typical time-scale for these solutions to be of order $M_{5}^{-1}$, so without significant fine-tuning of the solution the period of acceptable evolution will be too short. Even if the Friedmann equation does have appropriate late-time behaviour, there is no guarantee that the effective gravitational interactions of particles on the brane will take the standard form. By considering the effective four-dimensional gravity, we will be able to further constrain $W$. However in this paper I will concentrate on the implications of the non-standard Friedmann equation.

It is worth noting that (4.1 4.3) still satisfy equations (3.4) and (3.8) if we change the signs of $\mathcal{F}$ and $V$. In this case (4.4) becomes

$$
\frac{\dot{a}_{0}^{2}}{a_{0}^{2}}=\frac{\kappa^{4}}{36}\left[W_{0}^{2}+\rho_{\mathrm{b}}^{2}\right] .
$$


This can also be rewritten as (4.9), but with $\left(\lambda^{2}-1\right)$ replaced with $\left(\lambda^{2}+1\right)$. Thus these alternative solutions will not give the correct late time behaviour, because of the large effective cosmological constant term.

\section{Solutions for Exponential Superpotentials}

As an illustration I will consider superpotentials which are the sum of two exponentials. These are well motivated by string/M-theory. A time-independent example of one of them is discussed in ref. [9]. With the aid of the transformation $\phi \rightarrow \pm \phi+$ const. they can all be reduced to

$$
W=c\left[\frac{e^{-\alpha_{1} \phi}}{\alpha_{1}}+s \frac{e^{\alpha_{2} \phi}}{\alpha_{2}}\right]
$$

with $s= \pm 1$ and $\alpha_{1} \geq\left|\alpha_{2}\right|$, or if $\alpha_{2}=0$,

$$
W=c\left[\frac{e^{-\alpha_{1} \phi}}{\alpha_{1}}+\frac{s}{\kappa}\right] \text {. }
$$

Since the above superpotentials are loosely inspired by string theory, it is natural for the coefficients $\alpha_{i}$ to be of order $\kappa$, as in ref. [9].

If $s=+1$ and $\alpha_{2} \neq 0$ then $W$ has a minimum at $\phi=0$. If $s \alpha_{2}<0$ then $W$ has a zero at $\phi=\phi_{\star}=\left(\alpha_{1}+\alpha_{2}\right)^{-1} \ln \left(-s \alpha_{2} / \alpha_{1}\right)$, or $\phi_{\star}=-\alpha_{1}^{-1} \ln \alpha_{1}$ if $s=-1$ and $\alpha_{2}=0$.

The corresponding potentials obtained from (4.1) are

$$
V=\frac{c^{2}}{8}\left[\left(1-\frac{4 \kappa^{2}}{3 \alpha_{1}^{2}}\right) e^{-2 \alpha_{1} \phi}+\left(1-\frac{4 \kappa^{2}}{3 \alpha_{2}^{2}}\right) e^{2 \alpha_{2} \phi}-2 s\left(1+\frac{4 \kappa^{2}}{3 \alpha_{1} \alpha_{2}}\right) e^{\left(\alpha_{2}-\alpha_{1}\right) \phi}\right],
$$

and (for $\alpha_{2}=0$ )

$$
V=\frac{c^{2}}{8}\left[\left(1-\frac{4 \kappa^{2}}{3 \alpha_{1}^{2}}\right) e^{-2 \alpha_{1} \phi}-2 s \frac{4 \kappa}{3 \alpha_{1}} e^{-\alpha_{1} \phi}-\frac{4}{3}\right] .
$$

If $V$ is to be bounded from below then only some values of the parameters are allowed.

The solutions of (4.3) when $W$ is given by (5.1) are

$$
\ln \left(a / a_{*}\right)=-\frac{\kappa^{2}}{3 \alpha_{1} \alpha_{2}} \ln \left|e^{\alpha_{1} \phi}-s e^{-\alpha_{2} \phi}\right|
$$

where $a_{*}$ is an arbitrary constant.

When $\alpha_{2}=0$, we will use the superpotential (5.2) instead. In this case (4.3) is solved by

$$
\ln \left(a / a_{*}\right)=\frac{\kappa}{3 \alpha_{1}}\left(\kappa \phi+s e^{\alpha_{1} \phi}\right)
$$

The evolution of each of these solutions falls in to one of six cases, one for each choice of $s$ and the sign of $\alpha_{2}$. Since we are trying to produce a big bang style cosmology, we will be interested in solutions which start at $a=0$. There are also some solutions with $\phi=$ const. and $d W / d \phi=0$, but these are effectively the same as the constant bulk energy case (3.5), so I will not consider them further. 


\subsection{Superpotential with a minimum, $\alpha_{2}>0, s=+1$}

The expression (5.5) gives two solutions in this case. As $a$ goes from 0 to $\infty, \phi$ rolls down from either $+\infty$ or $-\infty$ to the minimum of the superpotential at $\phi=0$. While it is rolling down, $\dot{W} / W<0$, and so (4.7) implies that energy leaks off the brane.

The approximate behaviour of $\phi$ and $\mathcal{F}$ in terms of $a$ can be found when $a$ is small or large by using series and asymptotic expansions. Initially, when $a \approx 0$,

$$
\begin{gathered}
\phi \approx \operatorname{sgn}(\phi) \frac{3 \alpha_{i}}{\kappa^{2}} \ln \left(a_{*} / a\right), \\
\mathcal{F} \approx \frac{\kappa^{4} c^{2}}{36 \alpha_{i}^{2}}\left(\frac{a_{*}}{a}\right)^{6 \alpha_{i}^{2} / \kappa^{2}} .
\end{gathered}
$$

where the index $i$ is equal to 1 if $\phi<0$ and 2 if $\phi>0$. Substituting these expressions into (4.9), we find that $a \propto t^{1 /\left(4+3 \alpha_{i}^{2} / \kappa^{2}\right)}$. The initial expansion is thus faster than in the usual brane cosmology.

Later, when $a$ is large,

$$
\begin{gathered}
\phi \approx \frac{1}{\alpha_{1}+\alpha_{2}}\left(\frac{a_{*}}{a}\right)^{3 \alpha_{1} \alpha_{2} / \kappa^{2}}, \\
\mathcal{F} \approx \frac{\kappa^{4} c^{2}}{36 \alpha_{1}^{2} \alpha_{2}^{2}}\left[\left(\alpha_{1}+\alpha_{2}\right)^{2}+\alpha_{1} \alpha_{2}\left(\frac{a_{*}}{a}\right)^{6 \alpha_{1} \alpha_{2} / \kappa^{2}}\right] .
\end{gathered}
$$

Thus when $t$ (and hence $a$ ) is large, $W^{2} \rightarrow$ const. and so the standard cosmology will be obtained from (4.9), with order $\rho a_{0}^{6 \alpha_{1} \alpha_{2} / \kappa^{2}}$ corrections to the Friedmann equation. Since $\dot{W} \rightarrow 0$ at late time, the amount of energy leakage from the brane also tends to zero, as is required to agree with the standard cosmology.

\subsection{Superpotential with a zero, $\alpha_{2}>0, s=-1$}

In this case $\phi$ rolls from either $+\infty$ to $-\infty$, or vice versa. At both ends of the solution $a \rightarrow 0$. We see from (5.5) that $a$ is bounded.

Initially $\dot{W} / W$ is negative, so energy drains off the brane [see (4.7)]. This continues until $\phi=\phi_{\star}$, where $W=0$. At this point there is nothing left on the brane. This is also the point at which the scale factor $a$ reaches its maximum value $\left(a_{\max }\right)$. After

this the universe re-collapses. $\dot{W} / W$ is now positive, so energy is sucked onto the brane. This continues until $|\phi|$ and $|W|$ reach infinity, at which point $a$ has returned to 0 .

The approximate behaviours of $\phi$ and $\mathcal{F}$ near $a=0$ are given by (5.7) and (5.8). Near $a=a_{\max }$

$$
\begin{gathered}
\ln \left(a / a_{\max }\right) \approx-\frac{\kappa^{2}}{6}\left(\phi-\phi_{\star}\right)^{2} \\
\mathcal{F} \approx \frac{\kappa^{2} c^{2} e^{-2 \alpha_{1} \phi_{\star}}}{6 \alpha_{1}^{2}}\left(\alpha_{1}+\alpha_{2}\right)^{2} \ln \left(a_{\max } / a\right) .
\end{gathered}
$$


By substituting (5.8) and (5.12) into (4.9), a finite upper bound can found for the time taken to reach $a_{\max }$. While $\dot{a}=0$ there, (4.5) shows that $\dot{\phi} \neq 0$ and so $\phi$ continues to roll down (or up) the superpotential. Thus the universe expands to its maximum size and then re-collapses, all in finite time. For $\alpha_{i} \sim \kappa$, the time taken for the universe to re-collapse will be of order $M_{5}^{-1}$. This very short lifetime for the universe, and the way in which all energy (and hence matter) flows off the brane, mean that this model is completely incompatible with the standard cosmology.

The re-collapse of the universe is a common feature of solutions which pass through zeros of $W$. If $W$ changes sign and $d W / d \phi \neq 0$, then (4.3) implies that $d \phi_{0} / d a_{0}$ changes sign at this point. Equation (4.5) ensures that $\dot{\phi}_{0}$ does not, provided $\rho_{\mathrm{b}}^{2}>W_{0}^{2}$. Thus $\dot{a}_{0}$ must also change sign. This is consistent with the expression for $\dot{a}_{0}$, (4.4). Once $a_{0}$ starts to decrease, (4.4) implies that it will continue to do so.

\subsection{Positive monotonic superpotential, $\alpha_{2}<0, s=-1$}

There is just one solution for these parameters. The scalar field $\phi$ starts at $-\infty$ when $a=0$, and then rolls down to $+\infty$ as $a \rightarrow \infty$. The approximate large and small $a$ behaviour of $\phi$ and $\mathcal{F}$ are given by (5.7) and (5.8). While the initial behaviour of this solution is similar to that of section 5.1, $\mathcal{F} \rightarrow 0$ at late time. Thus the effective gravitational coupling tends to zero as $t \rightarrow \infty$.

This type of superpotential has been suggested as a possible candidate for quintessence [8]. If we set $\rho=0$ and $\lambda^{2} \neq 1$ in (4.9), we get a solution with a cosmological constant which decreases with time. Unfortunately when the matter fields are re-introduced, the solution is incompatible with the standard cosmology, due to the asymptotically vanishing gravitational coupling. Thus, this type of superpotential is not suitable after all, at least not for the cosmological generalisation of supergravity solutions discussed in this paper.

\subsection{Superpotential with minimum and zero, $\alpha_{2}<0, s=+1$}

For these parameters $a=0$ when $\phi=0$ and $\phi=-\infty$. This gives a total of three solutions. $\phi$ can roll from $-\infty$ to 0 , or vice versa. In both cases $a \rightarrow 0$ at the start and end of the solutions. These are similar to the solutions of section 5.2, in that the universe expands to a maximum value and then re-collapses. As before the maximum value is reached when $W=0$.

The other solution, with $\phi>0$, starts at $\phi=0$ and $a=0$. As $a$ increases to $\infty$, $\phi$ tends to $+\infty$. The approximate behaviour of $\phi$ and $\mathcal{F}$ there is given by (5.9) and (5.10) near $\phi=0$, and is asymptotically given by (5.7) and (5.8) when $|\phi|$ is large. Thus the $\phi>0$ solution has $W^{2} \rightarrow 0$ at large $t$ (and $a$ ), which is not cosmologically acceptable. 


\subsection{Special cases $\alpha_{2}=0, s= \pm 1$}

Asymptotic and series expansions of (5.6) give the approximate forms of $\phi$ and $\mathcal{F}$ for large and small $a$

$$
\begin{gathered}
\phi \approx\left\{\begin{array}{c}
\frac{3 \alpha_{1}}{\kappa^{2}} \ln \left(a / a_{*}\right) \quad \phi<0 \\
\frac{1}{\alpha_{1}} \ln \left[\frac{3 \alpha_{1} s}{\kappa} \ln \left(a / a_{*}\right)\right] \phi>0
\end{array}\right. \\
\mathcal{F} \approx \frac{\kappa^{2} c^{2}}{36}\left\{\begin{array}{l}
{\left[\frac{\kappa}{\alpha_{1}}\left(\frac{a_{*}}{a}\right)^{3 \alpha_{1}^{2} / \kappa^{2}}+s\right]^{2} \phi<0} \\
{\left[\frac{\kappa^{2}}{3 \alpha_{1}^{2} \ln \left(a / a_{*}\right)}+1\right]^{2} \phi>0}
\end{array}\right.
\end{gathered}
$$

Both these cases have $\phi$ rolling down the superpotential from $-\infty$ to $+\infty$. If $s=-1$ the system will pass through a zero of the superpotential, and as with the solution in section 5.2, $a$ reaches its maximum value there, and then starts to decrease.

The $s=+1$ case resembles the solution in section 5.1, in that $\mathcal{F} \rightarrow$ const. at late $t$ (large $a$ ). Thus this type of solution also provides a potentially viable cosmological model. The initial expansion of the universe is $a \propto t^{1 /\left(4+3 \alpha_{1}^{2} / \kappa^{2}\right)}$. The late time Friedmann equation resembles that of the usual brane cosmology with order $\rho / \ln a$ corrections.

\subsection{Summary}

The solutions considered in this section can be divided into two distinct categories. Firstly there are the solutions which pass through a zero of the superpotential. In this case the universe expands until $W=0$. Up until this point energy is lost from the brane into the bulk. By the time $W=0$ there is nothing left on the brane. After this the universe contracts, and energy flows back onto the brane. This continues until the universe re-collapses to $a=0$. This scenario appears to have little resemblance

to the standard cosmology. The solutions described in sec. 5.2, sec. 5.4 (first two cases), and sec. $5.5(s=-1)$ fall into this category.

Of course it is still possible that these solutions agree with the standard cosmology at some intermediate period of their evolution. However, unless the parameters are fine-tuned so that $\alpha_{i} / \kappa$ are extremely small, this period will be far to short to agree with observation.

From a cosmological point of view, the other solutions are more promising. In this case $\phi$ rolls down to a minimum of $|W|$, without passing through $W=0$. As the minimum is approached, $a \rightarrow \infty$. Energy also leaks off the brane in this case, but the rate approaches zero as $a \rightarrow \infty$. If the final value of $W$ is finite, then the standard cosmology can be obtained late in the evolution. The solutions in sec. 5.1 
and sec. 5.5 for $s=+1$ fall into this category, thus the only viable cosmological solutions with a superpotential of the form (5.1) or (5.2) are those with $s=+1$ and $\alpha_{2} \geq 0$. The solutions of sec. 5.3 and sec. 5.4 (third case) also have $\phi$ rolling down to a minimum, but since it has $W=0$ there, the energy remaining on the brane tends to zero as $a \rightarrow \infty$.

It should be noted that although the solutions with a minimum of $|W|$ give the correct Friedmann equation (and hence agree with many observations), their other properties could still conflict with experimental results, and so they may not be acceptable after all. For example, they may give the wrong effective gravitational forces for objects on the brane.

\section{Bulk solutions}

So far I have not determined the $y$ dependence of the metric (or $\phi$ ). Following ref. [3], this can be found by solving the non-zero off-diagonal component of the Einstein equations. When $\phi=\phi(a)$ this is

$$
G_{05}=3\left(\frac{n^{\prime} \dot{a}}{n a}+\frac{\dot{b} a^{\prime}}{b a}-\frac{\dot{a}^{\prime}}{a}\right)=\kappa^{2} T_{05}=\kappa^{2} a^{\prime} \dot{a}\left(\frac{d \phi}{d a}\right)^{2} .
$$

We can assume that $b=1$, in which case (6.1) is solved by

$$
\frac{\dot{a}}{n}=\beta(t) \frac{\kappa^{2}}{6} \exp \left\{-\frac{\kappa^{2}}{3} \int a\left(\frac{d \phi}{d a}\right)^{2} d a\right\}
$$

where the function $\beta$ is independent of $y$. If $V$ has a supergravity-like form (4.1), the above expression simplifies to

$$
\frac{\dot{a}}{n}=\beta(t) \frac{\kappa^{2}}{6} W(\phi) .
$$

Since we want $n_{0}=1$, (4.4) implies that $\beta=a_{0} \sqrt{\rho_{\mathrm{b}}^{2} / W_{0}^{2}-1}$.

Substituting (6.3) into the definition of $\mathcal{F}(3.1)$

$$
\left(a^{\prime}\right)^{2}=\frac{\kappa^{4}}{36}\left(\beta^{2}+a^{2}\right) W^{2}(\phi)
$$

$a$ must also satisfy $a=a_{0}$ at $y=0$ and the jump condition (2.10). Since we are interested in a $Z_{2}$ symmetric solution, $a$ will be a function of $|y|$.

The alternative solutions discussed at the end of section 1 satisfy

$$
\left(a^{\prime}\right)^{2}=\frac{\kappa^{4}}{36}\left(\beta^{2}-a^{2}\right) W^{2}(\phi)
$$

instead of (6.4). Solutions of this equation will have $a$ bounded in the bulk. Since they do not have the correct late time cosmological evolution, I will not consider them further. 
The corresponding time-independent bulk solutions can be obtained by setting $\rho_{\mathrm{b}}=W_{0}$, so that $\beta=0$. Finding analytic solutions of (6.4) is not generally possible, although solutions for a few special cases can be found.

If we take $\alpha_{1}=\alpha_{2}=\kappa / \sqrt{3}$ then the superpotential used in section 5.1 simplifies to

$$
W=\frac{2 c}{\alpha_{1}} \cosh \left(\alpha_{1} \phi\right)=\frac{c \sqrt{3}}{\kappa}\left[\left(\frac{a_{*}}{a}\right)^{2}+4\right]^{1 / 2} .
$$

The bulk behaviour of $a$ is

$$
a^{2}=a_{0}^{2}\left[1-\frac{\kappa^{2} \rho_{\mathrm{b}}}{3 \mu} \sinh (\mu|y|)+\left(\frac{\rho_{\mathrm{b}}^{2}}{2 W_{0}^{2}}+\frac{\kappa^{4} W_{0}^{2}}{18 \mu^{2}}\right)(\cosh (\mu y)-1)\right],
$$

where $\mu=2 c \kappa / \sqrt{3}$. $a^{2}$ has zeros if $\rho_{\mathrm{b}}>W_{0}>3 \mu / \kappa^{2}$, which is true for cosmological solutions. The position of these singularities varies with time. Unfortunately $\phi^{\prime}, \dot{\phi}$ and $W$ are all singular at these points, and so $T_{A B}$ is too. Thus the singularities at $a=0$ are naked curvature singularities. This problem could be resolved if we have a compact bulk, and one or more other branes at suitable distances from our brane [2, 11]. This separation of the branes will then be time dependent. In order for this scenario to work, the other brane(s) must have negative energy densities, which may lead to instabilities.

The bulk behaviour for the model discussed in section 5.2 can also be found when $\alpha_{1}=\alpha_{2}=\kappa / \sqrt{3}$. This time the bulk solution of $a^{2}$ is given by (6.7), but with $\mu$ replaced by $i \mu$. As before there will be bulk singularities.

Finally, for $\alpha_{1}=-\alpha_{2}=\kappa / \sqrt{3}$, the bulk behaviour of the solution in section 5.3 is

$$
a^{2}=a_{0}^{2}\left(1-\frac{\kappa^{2}}{3} \rho_{\mathrm{b}}|y|+\frac{\kappa^{4}}{36} W_{0}^{2} y^{2}\right) .
$$

Again $a^{2}$ has zeros in the bulk when $\rho_{\mathrm{b}}>W_{0}$, and since $T_{A B}$ is also divergent there, they are curvature singularities. In this case $W \propto 1 / a$ so the singularities occur when $|W| \rightarrow \infty$. This is also the case with the other two examples above. This suggests that solutions which have $W$ bounded may be curvature singularity free.

\section{Conclusions}

By considering solutions of the special form $\phi=\phi(a)$ it is possible to significantly simplify the field equations for a brane world cosmology with a scalar field. Furthermore, if the theory has a supergravity style potential, the simplified equations can be further reduced to three first order differential equations. This gives a generalisation of time-independent supergravity brane worlds to brane cosmologies. For simplicity I have considered solutions with $Z_{2}$ symmetric bulks.

Investigation of examples of these solutions suggests that the evolution of these brane cosmologies starts and ends at points with either $d W / d \phi=0$ or $W= \pm \infty$ ( $W$ 
being the superpotential). If the solution passes through a point with $W=0$, the universe will stop expanding, and re-collapse. The only solutions which avoid this re-collapse are those where $\phi$ rolls down to a minimum of $|W|$.

In order for these $Z_{2}$ symmetric, supergravity solutions to be self-consistent, the matter fields on the brane must couple to the bulk scalar in such a way that energy is not conserved on the brane. In the case of solutions which approach a minimum of $|W|$, the amount of leakage tends to zero, and the standard cosmology is obtained at late times. With the other solutions, all the energy will have leaked off by the time $W=0$ is reached. After this energy flows back onto the brane until it re-collapses.

The bulk behaviour of the metric (and $\phi$ ) can also be determined. Unfortunately for the exponential superpotentials considered in this paper, they all have naked curvature singularities in the bulk. The problems occur at points where $W= \pm \infty$. This suggests that a solution which only passes through finite values of $W$ may not have naked bulk singularities. The singularity problem could also be resolved by adding additional branes to the bulk. Like the singularities, the position of the branes would vary with time.

\section{Acknowledgements}

I wish to thank W. B. Perkins for useful discussions, and PPARC for financial support.

\section{References}

[1] L. Randall and R. Sundrum, A large mass hierarchy from a small extra dimension, Phys. Rev. Lett. 83 (1999) 3370 hep-ph/9905221]; An alternative to compactification, Phys. Rev. Lett. 83 (1999) 4690 hep-th/9906064.

[2] P. Binétruy, C. Deffayet, D. Langlois, Non-conventional cosmology from a braneuniverse, Nucl. Phys. B 565 (2000) 269 [hep-th/9905012].

[3] P. Binétruy, C. Deffayet, U. Ellwanger and D. Langlois, Brane cosmological evolution in a bulk with cosmological constant, Phys. Lett. B 477 (2000) 285 hep-th/9910219.

[4] H. A. Chamblin and H. S. Reall, Dynamic dilatonic domain walls, Nucl. Phys. B 562 (1999) 133 hep-th/9903225.

[5] T. Shiromizu, K. i. Maeda and M. Sasaki, The Einstein equations on the 3-brane world, Phys. Rev. D 62 (2000) 024012 [gr-qc/9910076].

[6] K. i. Maeda and D. Wands, Dilaton-gravity on the brane, Phys. Rev. D 62 (2000) 124009 hep-th/0008188;

A. Mennim and R. A. Battye, Cosmological expansion on a dilatonic brane-world, Class. and Quant. Grav. 18 (2001) 2171 hep-th/0008192. 
[7] C. van de Bruck, M. Dorca, C. J. Martins and M. Parry, Cosmological consequences of the brane/bulk interaction, Phys. Lett. B 495 (2000) 183 hep-th/0009056.

[8] P. Brax and A. C. Davis, Cosmological solutions of supergravity in singular spaces, Phys. Lett. B 497 (2001) 289 hep-th/0011045; Cosmological evolution on selftuned branes and the cosmological constant, J. High Energy Phys. 0105 (2001) 007 hep-th/0104023.

[9] K. Skenderis and P. K. Townsend, Gravitational stability and renormalization-group flow, Phys. Lett. B 468 (1999) 46 hep-th/9909070.

[10] A. Chamblin and G. W. Gibbons, Nonlinear supergravity on a brane without compactification, Phys. Rev. Lett. 84 (2000) 1090 hep-th/9909130;

O. DeWolfe, D. Z. Freedman, S. S. Gubser and A. Karch, Modeling the fifth dimension with scalars and gravity, Phys. Rev. D 62 (2000) 046008 hep-th/9909134;

C. Csaki, J. Erlich, C. Grojean and T. Hollowood, General properties of the self-tuning domain wall approach to the cosmological constant problem, Nucl. Phys. B 584 (2000) 359 hep-th/0004133.

[11] P. Brax and A. C. Davis, On brane cosmology and naked singularities, Phys. Lett. B 513 (2001) 156 hep-th/0105269. 